\title{
Time Sharing Policy in Wireless Networks for Variable Rate Transmission
}

\author{
Xiaolu Zhang, Meixia Tao and Chun Sum Ng \\ Department of Electrical and Computer Engineering \\ National University of Singapore \\ Singapore 117576 \\ Email: \{zhangxiaolu, mxtao, elengcs\}@nus.edu.sg.
}

\begin{abstract}
For most of wireless services with variable rate transmission, both average rate and rate oscillation are important performance metrics. One often needs to decide how much rate oscillation the service can tolerate to obtain a higher average rate. Service satisfaction for each user is quantified by an increasing and concave utility function of instantaneous transmission rate. It is capable of facilitating the resource allocation with flexible combinations of average rate and rate oscillation. Particularly, we are interested in maximizing the time-average aggregate utility by scheduling user transmissions in a time-shared wireless network. A resource allocation policy is developed, namely, time sharing (TS), to exploit the concavity of utility function and the fluctuation of channel gain. This is formulated as a constrained convex optimization problem. Our analysis shows that in the TS policy the optimal scheduler allows multiple users with relatively better channel conditions to share a same time frame in an adaptive time-division manner. In addition, the more concave the utility function is, the higher the probability of time frame sharing is. An extension to quantized time sharing with limited channel feedback (QTSL) for practical systems is all studied. Simulation results show that, two to three bits of channel state information (CSI) are sufficient for the performance of QTSL scheme to approach that of the optimal TS policy when the number of time slots in a time frame is not less than the number of users, especially, in high SNR region.
\end{abstract}

\section{INTRODUCTION}

Recently, resource allocation has been identified as a promising approach supporting diverse quality of service (QoS) requirement for wireless data service. To model the degree of user satisfaction with service quality, we resort to the concept of utility function. The utility can be constructed as a function of any given network performance metric, such as rate and delay.

Related work on utility-based wireless resource allocation can be found in [1]-[7]. In [7] and [2], the objective is to maximize a concave utility function of average transmission rate, and it is shown that the conventional proportional fair (PF) criterion is a special case where the utility is a $\log$ function of the rate. Considering the multi-user CDMA high data rate system with multiple-input multiple-output (MIMO) channels, a channel-dependent downlink scheduling scheme, named Alpha-Rule, is proposed [2]. Alpha-Rule enables flexible tradeoff between aggregate throughput and fairness by adjusting the parameter $\alpha$ in the utility function. The objective function (concave utility function of average transmission rate) in the above cases is only suitable for best-effort service since it could result in high rate oscillation.

Best-effort service may be viewed an extreme of rateoscillation sensitivity. Services such as audio and video applications generally have fairly low tolerance for rate oscillation. These services motivates the search for a new criterion that can be used to facilitate the choice of the combinations of average rate and rate oscillation.

In this paper, we propose a new network objective function, namely, Time-average Aggregate Utility of instantaneous Rate (TAUR). The proposed TAUR follows the following two rules: (1) When the average transmission rate is fixed, a large value of TAUR corresponds to small rate variation, and vice versa; (2) When the variation of the transmission rate is fixed, TAUR increases monotonically with the average transmission rate (based on Jensen inequality). As an illustrative example, we consider two cases. For case 1, the data stream is transmitted at a constant speed of $1 \mathrm{Mbit} / \mathrm{s}$ during the interval of 10 seconds. For case 2, no data is transmitted in the first 9 seconds and $10 \mathrm{Mbit} / \mathrm{s}$ is used for transmission in the last second. Obviously, the utilities of the two cases are identical as a function of average transmission rate. However, the time-average concave utility as a function of instantaneous transmission rate for case 1 is higher than that for case 2 , which is expected if the degree of user satisfaction is concerned. Therefore, TAUR is chosen here as the optimization objective.

The TAUR-based resource allocation problems are studied in a multi-user time-framed wireless system. At any instant only one user can transmit, but each time frame can be accessed by two or more users in an adaptive time-division fashion, depending on the channel conditions and system preferences. We intend to address the three challenges in wireless cellular environment [8]: limited radio resources, time-varying wireless channels, and limited battery power supply of mobile terminals. We first consider the optimal time sharing (TS) policy when perfect CSI is available at the base station. For a strictly concave utility function, our analysis shows that the time sharing policy allows users with relatively better channel conditions to share a same time frame so as to exploit both the concavity of utility function and the fluctuation of channel conditions. Specifically, all users in the network can transmit in a same time frame according to their associated time sharing 
fractions when the difference between their channel gains is insignificant. It is observed that, for a given number of users in a wireless network, the more concave the utility function is, the higher the time frame sharing probability is. The proposed TS resource allocation policy is formulated as a constrained convex variational optimization problem.

In practice, perfect channel feedback is not feasible due to limited capacity of feedback links. In addition, the possible time sharing fractions can only be chosen from a finite set of values considering switching latency and rigid synchronization requirement. To approach the performance of the optimal TS policy, a Quantized TS policy with Limited feedback (QTSL) is proposed. Interestingly, it is observed that when the number of quantized time-sharing slots per time frame is not less than the number of users, two to three bits of CSI are sufficient for the QTSL scheme to achieve a performance close to that of the optimal TS policy. When the number of quantized slots in a time frame is less than the number of users, the QTSL cannot fully exploit the concavity of utility function, but its performance improvement is still significant when compared to other policies such as MAX-RATE and Round-Robin. In addition, the numerical result shows that the performance gain obtained by increasing the number of feedback bits beyond two is very limited.

The rest of the paper is organized as follows. The system model is described in section II. Section III presents the optimal TS policy. In Section IV, QTSL policy is proposed by taking into account the limited channel feedback and quantized time sharing fraction. Numerical results and conclusions are provided in Section V and VI, respectively.

\section{SySTEM MODEL}

We consider a single cell consisting of $N$ mobile users communicating with a common base station. The base station centrally coordinates both the uplink and downlink transmissions on a time frame basis. The communication link between each user and the base station is modelled as a time-varying fading channel with additive white Gaussian noise (AWGN). The channel gains remain approximately unchanged during each transmission frame, but can vary from one frame to another. Only one user can access the channel at any time instant. At any given time frame, let the instantaneous channel gain of user $i$ be denoted as $g_{i}$ and follow a probability density function (PDF) $f_{i}(g)$, for $1 \leq i \leq N$. If the channels of different users are independent, the joint PDF of the channel gain vector $\mathbf{g}=\left[g_{1}, g_{2}, \ldots, g_{N}\right]$ is given by $f(\mathbf{g})=\prod_{i=1}^{N} f_{i}\left(g_{i}\right)$. For frequency-flat Rayleigh fading, each $g_{i}$ follows the exponential distribution:

$$
f_{i}(g)=\left\{\begin{array}{ll}
\frac{1}{\bar{g}_{i}} \exp \left(-\frac{g}{\bar{g}_{i}}\right) & g \geq 0 \\
0 & \text { otherwise }
\end{array},\right.
$$

where $\bar{g}_{i}$ is the average channel gain of user $i$. Let $p_{i}$ denote the transmit power allocated to or from user $i$. The achievable instantaneous transmission rate of user $i$ in the absence of other users' interference can be expressed as [9]

$$
c_{i}=\log _{2}\left(1+\frac{p_{i} g_{i}}{\beta N_{0}}\right),
$$

where $N_{0}$ is the noise power, and $\beta$ is the signal-to-noise ratio (SNR) gap [10]. When instantaneous mutual information is used to characterize the achievable transmission rate, we have $\beta=1(0 \mathrm{~dB})$. If practical signal constellations are used, $\beta$ is a constant related to a given bit-error-rate (BER) constraint. For example, when uncoded QAM constellation is used, we have $\beta=-\ln (5 \cdot \mathrm{BER}) / 1.5$. We assume that each time frame can be accessed by all the $N$ users in an adaptive time-sharing fashion. Let $\boldsymbol{\rho}(\mathbf{g})=\left[\rho_{1}(\mathbf{g}), \rho_{2}(\mathbf{g}), \ldots, \rho_{N}(\mathbf{g})\right]$ denote the time-sharing adaptation policy, where $\rho_{i}$ represents the fraction of the frame duration allocated to user $i$. Thus, the actual transmission rate $r_{i}$ and the achievable transmission rate $c_{i}$ are related as $r_{i}=\rho_{i}(\mathbf{g}) c_{i}$. Here, we assume that each user is aware of its own channel gain, $g_{i}$, and the base station has the knowledge of the channel gains from all users, g, at the start of each time frame.

The utility considered in this paper is a function of the instantaneous transmission rate. When user $i$ is allocated with a fraction, $\rho_{i}$, of the given time frame, the user's utility is denoted as $U_{i}\left(r_{i}\right)$. The exact expression for the utility function $U_{i}(\cdot)$ is not crucial. Our analysis throughout this paper is valid for any utility function that is increasing, differentiable and concave. This also agrees with the utility function for Internet data addressed by Shenker in [11]. The form of $U_{i}(\cdot)$ depends on the user's specified service, and its parameters can be determined through sophisticated subjective surveys [8].

\section{OPTIMAL TIME SHARING}

In this section, we assume that the transmit powers are identical and time-invariant, i.e., $p_{i}=p, \forall i$, and that the wireless network is fully loaded. We choose the aggregate utility, which is the sum of individual user utilities, as the performance measure. The goal is to find an optimal timesharing adaptation policy $\rho^{*}(\mathrm{~g})$ relative to the instantaneous channel conditions $\mathrm{g}$ so as to maximize the TAUR of the system. Since the channel processes are ergodic, the optimization problem can be expressed mathematically as

$$
\begin{aligned}
\max _{\boldsymbol{\rho}(\mathbf{g})} I_{\mathrm{TS}} & \triangleq \mathbb{E}\left[\sum_{i=1}^{N} U_{i}\left(r_{i}\right)\right] \\
& =\int_{\mathbf{g}} \sum_{i=1}^{N} U_{i}\left(\rho_{i}(\mathbf{g}), g_{i}\right) f(\mathbf{g}) \mathbf{d} \mathbf{g},
\end{aligned}
$$

The constraints arise from the fact that all the users must compete for the limited resources available. According to the definition of $\rho_{i}(\mathbf{g})$ in Section II, the time sharing vector satisfies

$$
0 \leq \rho_{i}(\mathbf{g}) \leq 1, \text { for } i=1,2, \ldots, N
$$

and

$$
\sum_{i=1}^{N} \rho_{i}(\mathbf{g})=1
$$




\section{A. General Solution}

Since the constraints (4) and (5) are defined for all channel states, the time-average aggregate utility maximization in (3) is equivalent to maximizing instantaneous aggregate utility for each channel state. By applying the Lagrange equation, the optimal solution, $\rho_{i}^{*}$ can be expressed as

$$
\rho_{i}^{*}(\mathbf{g})=\left[\left(\frac{\partial U_{i}}{\partial \rho_{i}}\right)^{-1}\left(\lambda, g_{i}\right)\right]^{+} \text {for } i=1,2, \ldots, N \text {. }
$$

In (6), $\left(\frac{\partial U_{i}}{\partial \rho_{i}}\right)^{-1}\left(\cdot, g_{i}\right)$ is the inverse function of $\frac{\partial U_{i}}{\partial \rho_{i}}\left(\cdot, g_{i}\right)$ with respect to $\rho_{i}{ }^{1},(x)^{+} \triangleq \max (0, x)$, and $\lambda$ can be determined by substituting $\rho_{i}^{*}$ into the constraints (5). As can be seen, the optimal time-sharing policy is only a function of the instantaneous channel conditions and is independent of the channel statistics, although the objective is to maximize the average aggregate utility over the channel statistics.

In the next section, we consider a specific utility function and discuss the interesting properties of the optimal solution.

\section{B. Solution for log Utility Function}

Intuitively, the utility function, $U(r)$, should follow the law of diminishing return with $U(0)=0$. One example of such a function is

$$
U(r)=U(\rho c)=\ln \left(1+\frac{\rho c}{A}\right),
$$

where $A$ is a positive constant with the same unit as the achievable rate $c$. The quantity $A$ may be treated as a concavity parameter: a smaller $A$ corresponds to a more concave utility function, and vice versa. For the sake of simplicity, we assume that the utility function and PDF of channel gains are identical for all users, i.e., $U_{i}(\cdot)=U(\cdot)$ and $f_{i}(\cdot)=f_{0}(\cdot)$ for all $i$ 's. All the results obtained here can be easily generalized to the heterogeneous case.

The solution for the scenario with only two users is expressed as (8) at the top of the next page, and illustrated in Fig. 1, where $C_{i}=c_{i} / A$ is the scaled achievable transmission rate of user $i$. As Fig. 1 shows, the scaled achievable rate space $\left(C_{1}, C_{2}\right)$ for the two-user case is divided into three regions by the two curves $\frac{1}{C_{2}}-\frac{1}{C_{1}}=1$ and $\frac{1}{C_{2}}-\frac{1}{C_{1}}=-1$. Only one user is scheduled for transmission in regions 1 and 3 , whereas both users can transmit in region 2 . Also, $\rho_{i}^{*}$ increases monotonically as $g_{i}$ in region 2 . Noticing that, since the achievable transmission rate $c_{i}$ is a monotonically increasing function of the channel gain $g_{i}$ as in (2), the achievable transmission rate depends on the instantaneous channel conditions. We draw the following conclusion from this example. To maximize the system average aggregate utility, the two users should transmit in a same time frame when the difference between their instantaneous channel gains is insignificant. Moreover, the user with relatively better channel condition should have a larger fraction of time usage. Conversely, when the instantaneous channel gain of one user differs significantly from the other,

\footnotetext{
${ }^{1}$ Because the utility function is concave in $\rho, \frac{\partial U_{i}}{\partial \rho_{i}}\left(\cdot, g_{i}\right)$ is monotonously decreasing function of $\rho$. Therefore, the inverse function exists.
}

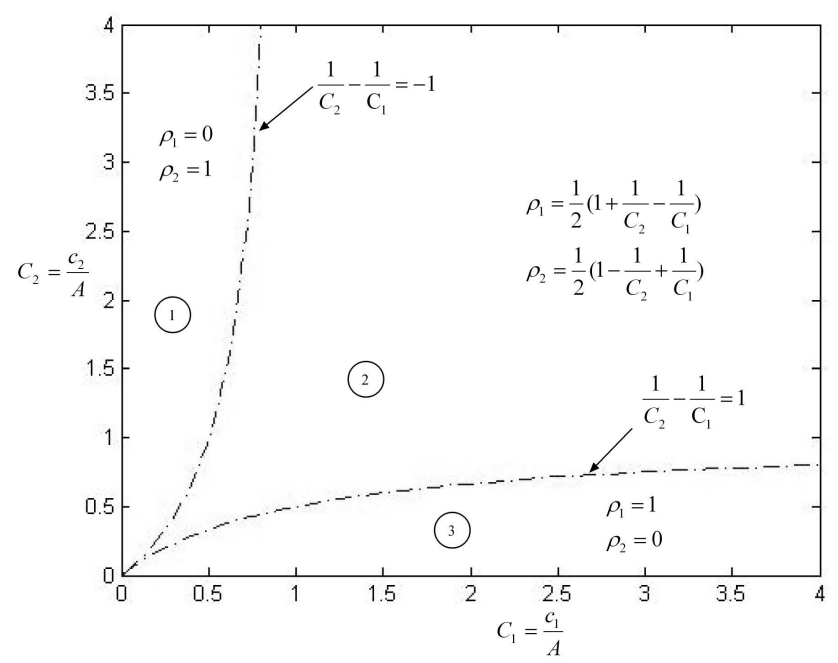

Fig. 1. Illustration of time sharing control vector for the case of two users

only the user with the better channel gain will transmit during whole time frame.

\section{Quantized Time Sharing With Limited Channel FEEDBACK}

The optimal time-sharing policy discussed in the previous section assumes that the CSI is perfectly known by the scheduler and that the time-sharing parameter can take arbitrary value between 0 and 1 . This provides an upper limit on the achievable performance (without power control) in the multiuser system considered. In this section, we shall investigate the effect of both partial channel feedback and discrete timesharing fractions.

For the downlink, the base station needs to know each user's CSI. This could be gained by sending the CSI from each user to the base sation through a feedback channel upon channel estimation at each user terminal. In practice, perfect channel feedback is not feasible due to limited capacity of the feedback links. To efficiently make use of the benefits brought by time sharing, the total time of feedback transmission should be much less than channel coherence time. We assume that the channel estimate of each user is quantized into $K=2^{M}$ regions, where $M$ is the number of feedback bits for each user. We partition channel gain into $K$ regions using a similar method in [12]. Let $\mathbb{G}=\left\{G_{1}, G_{2}, \ldots, G_{K+1}\right\}$ be the set of channel gain thresholds in increasing order with $G_{1}=0$ and $G_{K+1}=\infty$. If the channel gain of user $i$ falls in range $\left[G_{k}, G_{k+1}\right)$, we say user $i$ is in state $k$, and denote it as $S_{i}=k$. According to (1), the static probability of channel state $k$ is

$$
\Pi_{k}=\exp \left(\frac{-G_{k}}{\bar{g}}\right)-\exp \left(\frac{-G_{k+1}}{\bar{g}}\right) .
$$

If equal-probability method is used, i.e., $\Pi_{k}=1 / K$ for all $k$ 's, the channel gain thresholds can be determined from (9).

In practice, time sharing fractions cannot be an arbitrary number, but are restricted to a finite set of values due to 


$$
\begin{cases}\rho_{i}^{*}=\frac{1}{2}\left(1+\sum_{j=1}^{2} \frac{1}{C_{j}}\right)-\frac{1}{C_{i}}, & \text { if }-1 \leq \frac{1}{C_{2}}-\frac{1}{C_{1}} \leq 1 \quad \text { for } i=1,2 \\ \rho_{1}^{*}=1 \text { and } \rho_{2}^{*}=0, & \text { if } \frac{1}{C_{2}}-\frac{1}{C_{1}}>1 \\ \rho_{1}^{*}=0 \text { and } \rho_{2}^{*}=1, & \text { if } \frac{1}{C_{2}}-\frac{1}{C_{1}}<-1\end{cases}
$$

switching latency and difficulties in rigid synchronization. Therefore, we assume that a time frame can only be partitioned into $L$ sections with equal length. Each section is called as a time slot. Correspondingly, the number of users which can transmit in the same frame is limited by $L$. At the beginning of each frame, the base station computes the optimal timesharing policy $\rho^{*}$ defined in (10) at the top of the next page upon obtaining the channel states $\mathbf{S}=\left[S_{1}, S_{2}, \ldots, S_{N}\right]$ from all users.

The time sharing policy considered in this section maps the current channel states $\mathbf{S} \in \mathbb{R}_{+}^{N}$ to a time-sharing vector $\rho \in \mathbb{R}_{+}^{N}$. Intuitively, a codebook could be designed, and the base station can select the time sharing policy from the codebook. However, it can be seen that the total number of possible system states is $K^{N}$. This results in a highly complex solution to (10) for large $K$ and $N$. To avoid the complexity of $\mathcal{O}\left((K L)^{N}\right)$ in exhaustive search, an online greedy algorithm is proposed. This algorithm can obtain the optimal timesharing fractions $\rho^{*}$ with complexity $\mathcal{O}(L N)$. The greedy algorithm is carried out at the beginning of each time frame. The algorithm can be also called "marginal allocation". That is, beginning with an initial solution $\boldsymbol{\rho}=[0,0, \ldots, 0]$, one time slot is assigned at each iteration to the most favorable user (in the sense of maximizing the increment of the current objective value) until $\sum_{i} \rho_{i}=1$.

The description of greedy algorithm is as follows:

\section{1) Initialization}

Let $v=0$ ( $v$ is the index of the time slot to be allocated in a time frame), $\rho_{i}^{(0)}=0$ and $U_{i}^{(0)}=0$ for all $1 \leq i \leq$ $N$.

2) Allocate the $(v+1)^{\text {th }}$ time slot to user indexed by $i^{*}$, where $i^{*}$ satisfies (14) at the top of the next page.

Let $\rho_{i^{*}}^{(v+1)}=\rho_{i^{*}}^{(v)}+1 / L$ and $\rho_{i}^{(v+1)}=\rho_{i}^{(v)}$ for $i \neq i^{*}$.

3) Let $v=v+1$, and return to Step 2) until $v=L$

The optimal time sharing policy $\rho^{*}$ obtained as

$$
\boldsymbol{\rho}^{*}=\boldsymbol{\rho}^{(L)}
$$

Fig. 2 illustrates an example of quantized time sharing policy with limited feedback. Each time frame only consists of $L=2$ time slots and the number of feedback bits for CSI is $M=3$. When the average SNR is $3 \mathrm{~dB}$, the channel gains are partitioned into 8 states with equal probabilities. The corresponding thresholds are $0.13353,0.28768,0.47,0.69315$, $0.98083,1.3863$, and 2.0794, respectively. As a special case, the utility function has the same form as in (7) with $A=1$. Fig. 2 shows that if the channel state pair falls in the shaded area, the two users share the same frame with equal fraction, otherwise, the user with better channel state will occupy the whole frame.

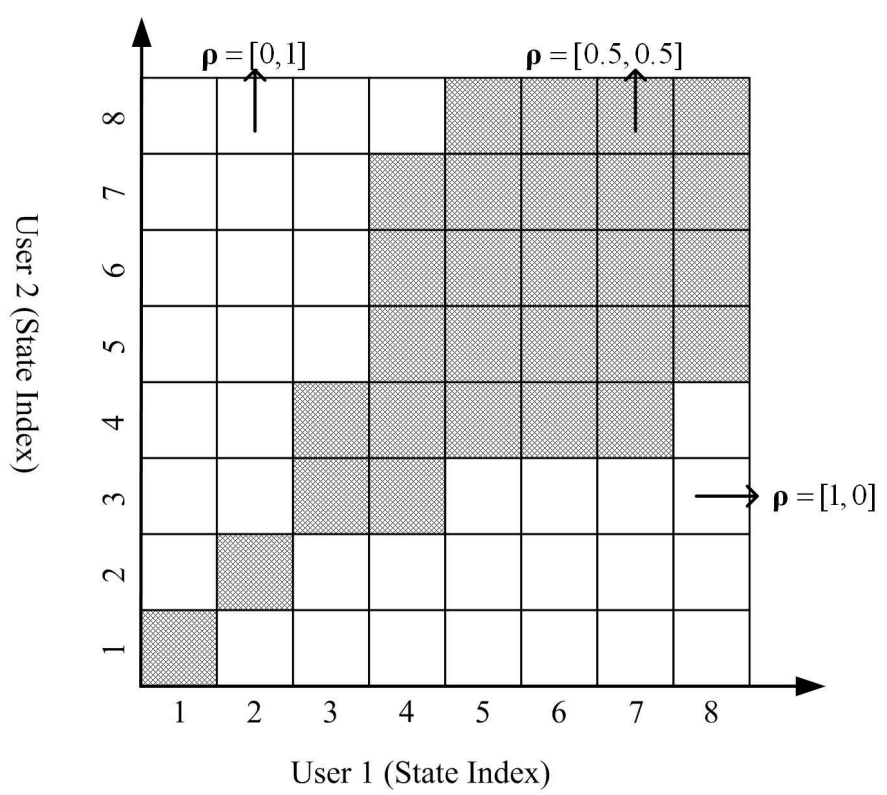

Fig. 2. Illustration of the quantized time sharing policy with limited feedback for two-user scenario with $L=2$ and $M=3$

\section{Numerical RESUlts AND Discussions}

In this section, the performance of the proposed TS and QTSL policies in a single cell system is evaluated using computer simulation. First, the relationship between the concavity of utility function and the time sharing probability is illustrated. Then, we demonstrate the impact of multiuser diversity on average aggregate utility and compare the performance of the optimal time sharing policy with that of other scheduling policies. Finally, the performance of the QTSL scheme is compared with that of the optimal TS policy.

The instantaneous channel gain between each user and the base station is generated using [13]

$$
g=\alpha d^{-n} 10^{0.1 \varsigma} X^{2}
$$

where $\alpha$ is a constant, $d$ is the distance between the user and the base station, $n$ is the path loss exponent, $10^{0.1 \varsigma}$ is the log-normal shadowing with $\varsigma$ being a Gaussian random variable, and $X$ is random variable with normalized Rayleigh distribution. We assume uncoded QAM with continuous rate adaptation is used. The target BER is set as $10^{-5}$, which results in a SNR gap of $8.2 \mathrm{~dB}$. The feedback channel is assumed to be error free and has no delay. Each run comprises 50,000 time frames.

We consider the homogeneous scenario, where all the users are located equidistant and far from the base station and have utility functions of the form given in (7) with identical 


$$
\begin{aligned}
\boldsymbol{\rho}^{*}(\mathbf{S})= & \underset{\boldsymbol{\rho}(\mathbf{S})}{\arg \max _{G_{S_{1}}}} \int_{G_{S_{2}}}^{G_{S_{1}+1}} \int_{G_{S_{N}}}^{G_{S_{2}+1}} \ldots \int_{i}^{G_{S_{N}+1}}\left[\sum_{i}^{N} U_{i}\left(\rho_{i}(\mathbf{g}), g_{i}\right)\right] \mathrm{d} \mathbf{g} \\
\text { s.t. } \quad & \rho_{i} \in\left\{0, \frac{1}{L}, \frac{2}{L}, \ldots, 1\right\}, \text { for } i=1,2, \ldots, N \\
& \sum_{i}^{N} \rho_{i}=1 .
\end{aligned}
$$

$$
i^{*}=\arg \max _{i \in\{1, \ldots, N\}} \int_{G_{S_{i}}}^{G_{S_{i}+1}}\left[U_{i}\left(\rho_{i}^{(v)}+1 / L\right)-U_{i}\left(\rho_{i}^{(v)}\right)\right] \mathrm{dg} .
$$

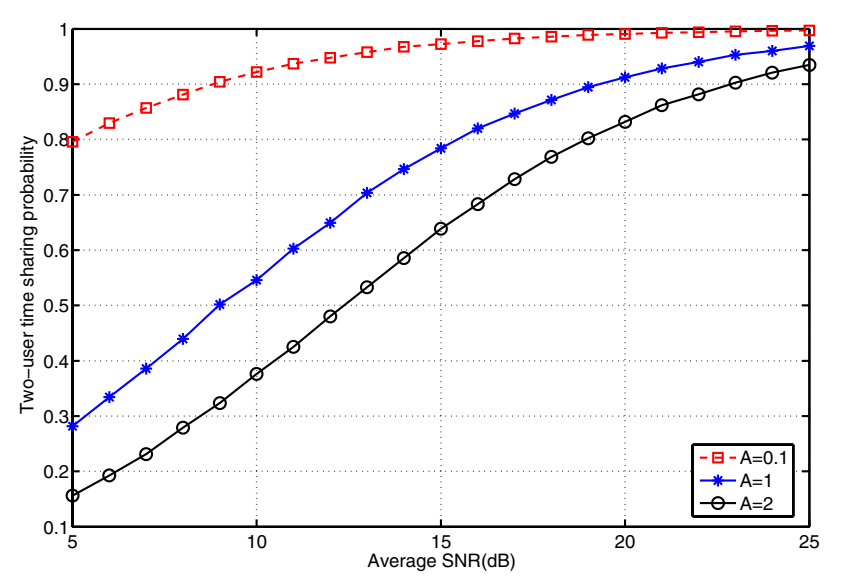

Fig. 3. Time frame sharing probability for two-user scenario

parameters. Fig. 3 shows the probability of time sharing with with $\rho_{i} \neq 0$ for all $i$ 's in a two-user network. When the average SNR increases, the probability that two users transmit in a same time frame increases. Furthermore, given a fixed average SNR, the more concave the utility function is ( $A$ becomes smaller), the higher the time sharing probability is. In particular, when $A=0.1$, the two users transmit in a same time frame with probability of 0.9 or higher if SNR $>10 \mathrm{~dB}$. In the remaining simulation, we let $A=0.1$.

Fig. 4 shows the average aggregate utility of the system by using different resource allocation policies, namely, the proposed TS, MAX-RATE and Round-Robin, at various average SNR's. The number of users varies from two to four. In the MAX-RATE policy, the scheduler always admits the user with the best channel gain to the channel in each time frame. In the Round-Robin policy, users are scheduled for transmission periodically, regardless of the channel condition. In Fig. 4, the performance curves of the Round-Robin policy for different users overlap. This is because Round-Robin does not exploit the multi-user diversity. The margin between the curves for 2, 3 and 4 users of MAX-RATE decreases with the increase of average SNR. On the contrary, the proposed TS policy can effectively exploit multi-user diversity where the diversity gain

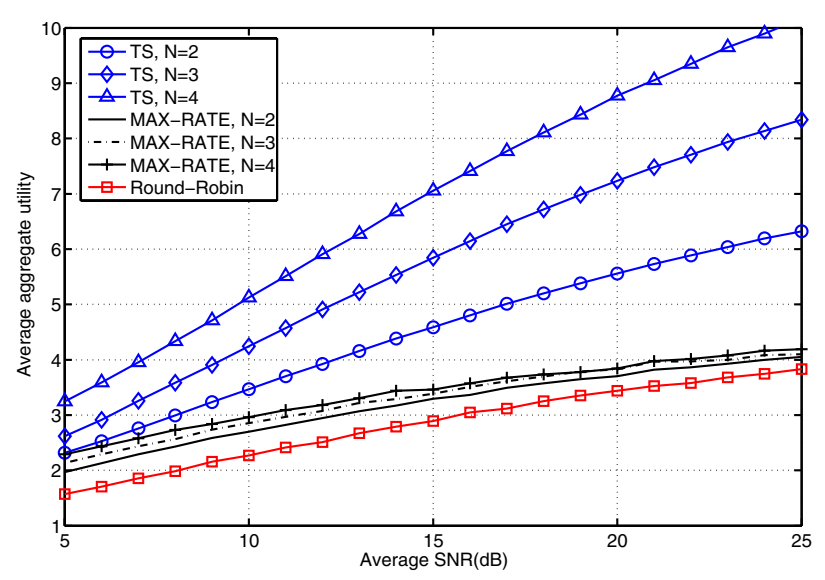

Fig. 4. Performance comparison of different resource allocation schemes

is observed to increase as the average SNR increases.

Fig. 5 compares the average rate and the standard deviation of rate for TS by varying the parameter $A$. the TS based on TAUR has a flexible balance between the average rate and the rate oscillation through adjusting the concavity of utility function.

Fig. 6 shows the performance of the quantized time sharing policy with limited channel feedback. The CSI is assumed to be quantized at 3-bit resolution. And the number of time slots is assumed to be the same as the number of users in the system. It is shown that the performance of the QTSL scheme approaches that obtained by the optimal TS policy, especially in high SNR region. Basically, the partition of time frame exploits the concavity of utility function; the multiuser diversity exploits the fluctuation of channel gains. When the number of time slots in a time frame is not less than the number of users, both these advantages of TS will be realized, i.e., the performance of the optimal TS policy can be approached.

In Fig. 7, we illustrate the performance of the QTSL scheme when the number of time slots in a time frame is less than the the number of users in the system. We consider $N=4$ users and vary the number of feedback bits from 1 to 3 , the 

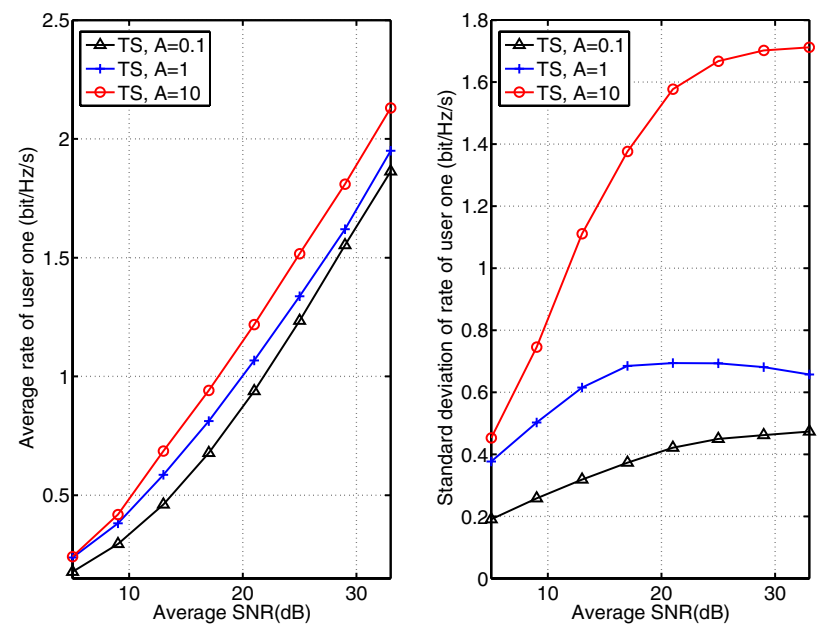

Fig. 5. average rate and standard deviation of rate versus average SNR using TS

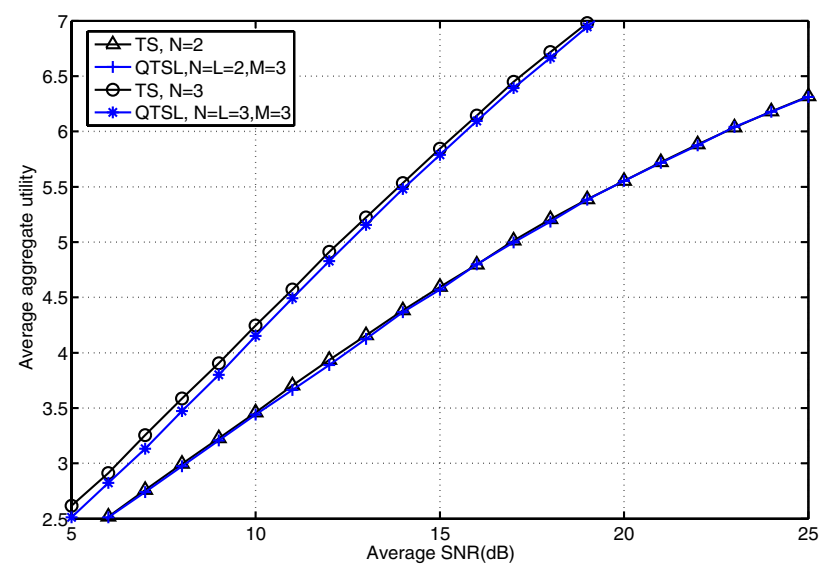

Fig. 6. Performance comparison of the optimal TS policy and QTSL policy for $N=2$ and 3 users, with $L=N$ slots and $M=3$ feedback bits

number of time slots in a frame from 2 to 3 . It is observed from Fig. 7 that there is a performance gap between QTSL schemes with $L<N$ and the optimal TS policy, but the average aggregate utility function with $N=4$ and $L=3$ is higher than that of the optimal TS policy with $N=3$. In addition, the performance gain begins to floor when the CSI is quantized beyond 2-bit resolution, i.e., $M \geq 2$.

\section{CONCLUSION}

In this paper, we develop a framework for wireless resource allocation based on maximum average aggregate utility. A time sharing policy is designed to exploit the fluctuation of the channel condition and the concavity of utility function. The proposed TS policy achieves significant performance improvement over the MAX-RATE policy. From the perspective of implementation, a QTSL scheme is developed. Simulation results show that two to three bits of CSI are sufficient for the

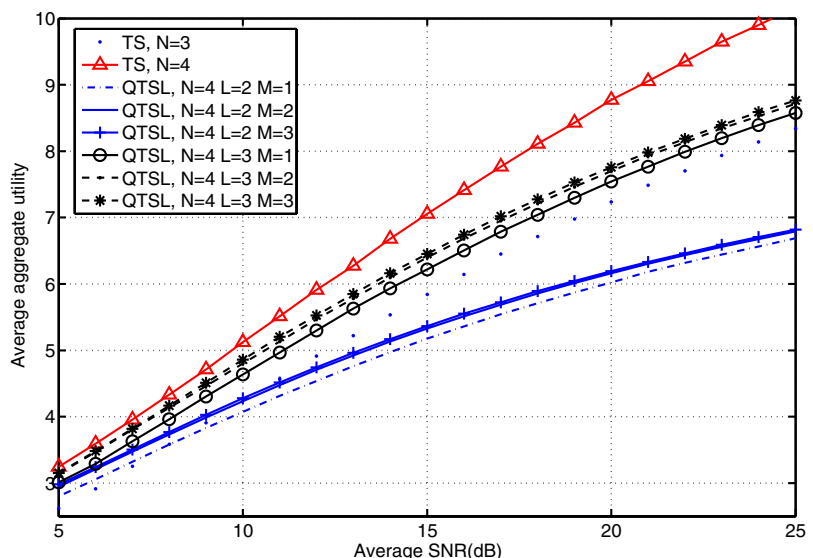

Fig. 7. Performance comparison of $N=4$ users, with $L=2$ and 3 time slots, and with $M=1,2$ and 3 feedback bits

QTSL scheme to realize most of the gains achievable by the optimal TS policy, when the number of time slots in a time frame is equal to or more than the number of users in the system.

\section{REFERENCES}

[1] Z. Jiang, Y. Ge, and Y. Li, "Max-utility wireless resource management for best-effort," IEEE Trans. on Wirel. Commun., vol. 4, no. 1, pp. 100111, Jan. 2005.

[2] A. Sang, X. Wang, M. Madihian, and R. D. Gitlin, "A flexible downlink scheduling scheme in cellular packet data systems," IEEE Trans. on Wirel. Commun., vol. 5, no. 3, pp. 568-577, March 2006.

[3] E. Yeh, "Delay-optimal rate allocation in multiaccess communications: a cross-layer view," in Proc. Multimedia Signal, 2002.

[4] M. Xiao, N. B. Shroff, and E. K. Chong, "A utility-based power control scheme in wireless cellular systems," IEEE/ACM Trans. on Networking, vol. 11, no. 2, pp. 210-221, April 2003.

[5] R. Berry, P. Liu, and M. Honig, "Design and analysis of downlink utility-based schedulers," in Proc. of the 40th Annual Allerton Conf. on Communication, Control, and Computing, 2002.

[6] F. Meshkati, H. V. Poor, S. C. Schwartz, and N. B. Mandayam, "An energy-efficient approach to power control and receiver design in wireless data networks," IEEE Trans. on Commun., vol. 53, no. 11, pp. 1885-1894, Nov. 2005.

[7] M. Andrews, L. Qian, and A. Stolyar, "Optimal utility based multi-user throughput allocation subject to throughput constraints," in Proc. IEEE INFOCOM '05, 2005.

[8] H. Jiang, W. Zhuang, X. Shen, and Q. Bi, "Quality-of-service provisioning and efficient resource utilization in cdma cellular communications," IEEE J. Select. Areas Commun., vol. 24, no. 1, pp. 4-15, Jan. 2006.

[9] X. Qiu and K. Chawla, "On the performance of adaptive modulation in cellular systems," IEEE Trans. on Commun., vol. 47, no. 6, pp. 884-895, Jun. 1999.

[10] A. J. Goldsmith and M. Effros, "The capacity region of broadcast channels with intersymbol interference and colored gaussian noise," IEEE Trans. on Infor. Theory, vol. 47, pp. 219-240, Jan. 2001.

[11] S. Shenker, "Fundamental design issues for the future internet," IEEE J. Select. Areas Commun., vol. 13, no. 7, pp. 1176-1188, Sep. 1995.

[12] Q. Zhang and S. A. Kassam, "Finite-state markov model for rayleigh fading channels," IEEE Trans. Commun., vol. 47, no. 11, pp. 1688-1692, Nov. 1999.

[13] T. S. Rappaport, Wireless Communications: Principles and Practice. Prentice Hall, 1999. 